14

\title{
Математическое моделирование влияния температуры на характер связывания мономерных белков в водных растворах
}

\author{
(C) Т.В. Кошлан, ${ }^{1}$ К.Г. Куликов ${ }^{2}$ \\ ${ }^{1}$ Санкт-Петербургский государственный университет, \\ 199034 Санкт-Петербург, Россия \\ ${ }^{2}$ Санкт-Петербургский политехнический университет Петра Великого, \\ 195251 Санкт-Петербург, Россия \\ e-mail: kulikov.kirill.g@gmail.com
}

(Поступило в Редакцию 23 марта 2017 г.)

Работа посвящена математическому моделированию исследования влияния температуры на стабильность гистоновых димеров Н2А-H2B и Н3-H4 путем изучения их поведения в различных температурных режимах от 20 до $50^{\circ} \mathrm{C}$. Изучение термической стабильности двух димеров в водных растворах при различных температурах выявило разнохарактерное поведение гистоновых димеров при температурах от 20 до $50^{\circ} \mathrm{C}$. Проведенный анализ показал, что димер Н3-Н4 выявляет склонность к агрегированию за счет увеличения силы связывания между гистонами Н3 и Н4. Исследования поведения гистонового димера Н2А-Н2B выявили различные температурные переходы в структуре гистонового димера с максимальным пиком на температуре $45^{\circ} \mathrm{C}$.

DOI: 10.21883/JTF.2017.11.45139.2256

\section{Введение}

Настоящая работа продолжает цикл работ [1-3] по исследованию поведения биологических комплексов in vitro на примере гистоновых димеров $\mathrm{H} 2 \mathrm{~A}-\mathrm{H} 2 \mathrm{~B}$ и H3-H4.

Разработан теоретический метод, позволяющий анализировать вклад различных участков полипептидной белковой цепи в образование и стабилизацию биологического комплекса, а также определять участки белковых молекул, отвечающих за агрегацию белков в водных растоворах при различных температурных режимах от 20 до $50^{\circ} \mathrm{C}$.

Отметим, что исследование проблемы агрегации белков представляет собой одно из актуальных направлений современной молекулярной биофизики. Агрегация белков является одним из процессов, который постоянно происходит в клетке. Каждый белок характеризуется своей нативной конформацией, пребывание в которой позволяет ему выполнять свои заданные биологические функции. Однако генетические мутации и ошибки при синтезе белков на рибосоме могут приводить к образованию неправильно свернутых белковых структур. Даже для нативных белков всегда существует вероятность частичного нарушения нативной структуры, особенно в условиях стресса (например, теплового, окислительного или осмотического). При нарушении нативной структуры белки перестают выполнять свои биологические функции, становятся менее стабильными и могут проявлять склонность к агрегированию, что может приводить к широкому спектру патологических состояний клетки и целого организма.

Таким образом, разработанный в предложенной работе подход позволит исследовать и объяснить патологиче- ские аспекты, связанные с изменением структуры белков в процессе агрегации.

Работа состоит из трех частей. В первой части мы опишем основные свойства белков и характер их поведения при повышении температуры. Вторая часть посвящена построению математической модели. В третьей части представлены численные результаты и графики проведенного моделирования. В заключении приведены основные выводы, полученные в работе.

\section{1. Основные свойства белков и характер их поведения при повышении температуры}

Известно, что незначительное повышение температуры может приводить как к агрегации одних белков, так и к растворению других белков. Рассмотрим более подробно гидрофобные взаимодействия, которые отвечают за агрегацию белков [4-6]. Ранее было выдвинуто предположение, что взаимодействия между гидрофобными аминокислотными остатками увеличиваются по мере увеличения температуры и уменьшаются по мере уменьшения температуры [5].

По мере того как аминокислотная последовательность принимает нативную структуру, гидрофобные остатки находятся в основном внутри глобулярной структуры белка, вдали от водного окружения. При повышении температуры происходит нарушение нативной структуры белка, что выражается в потере функций белка. Гидрофобные остатки выходят на поверхность молекулы и могут начать взаимодействовать с гидрофобными остатками других белков.

Существует несколько различных взаимодействий по своей природе, которые определяют структуру белка: 
водородные взаимодействия, гидрофобные взаимодействия, взаимодействия заряженных аминокислот, ковалентные связи между остатками цистеинов. В нативном состоянии молекула белка, как правило, является плотноупакованной, так что боковые группы, находящиеся во внутренней части молекулы, имеют ограниченную свободу движений. Особенно ограничено движение боковых групп, образующих гидрофобное ядро молекулы. Повышая температуру, можно создать такие внешние условия, при которых возрастают мелкомасштабные флуктуации отдельных групп атомов. После того как гидрофобные аминокислотные остатки выходят на поверхность и начинают взаимодействовать с другими гидрофобными аминокислотными остатками, белковая молекула денатурирует и при определенных условиях могут образовываться агрегаты (например, объединение гидрофобных частей молекулы).

Отметим, что денатурация - это разрушение нативной пространственной структуры молекулы белка, которое приводит к потере его биологической активности [6]. Следует отметить, что при повышении температуры молекулы белков не обязательно агрегируют, но могут также терять свою нативную структуру, не образовывая при этом агрегаты. Растворимость различного рода белков варьируется в широких пределах. Растворимость белка зависит от соотношения полярных и неполярных групп, их взаимного расположения и от результирующего дипольного момента. Большое количество полярных групп должно увеличивать как сродство белков к воде, так и их растворимость. Однако ионные группы могут оказать обратное действие, соединяясь с группировками противоположного знака и образуя внутри- и межмолекулярные солеобразные связи. Образование же таких межмолекулярных связей всегда ведет к дегидратации и способствует возникновению крупных нерастворимых агрегатов, поэтому денатурация белков также является функцией концентрации белка в растворе. В воде силы электростатического взаимодействия благодаря высокой диэлектрической проницаемости убывают, и возникают взаимодействия между полярными группами молекулы и воды. Если взаимодействие между белком и растворителем сильнее, чем между аминокислотами белка, то происходит растворение белка. Отметим, что водоудерживающая способность, как и растворимость, одновременно зависит от степени воздействия как белков с водой, так и белка с белком, так и от конформации и степени денатурации белка. Ввиду этого тепловая обработка оказывает сильное воздействие на эти физические свойства [7-9]. Во многих случаях тепловая обработка также снижает растворимость белков и может при определенных условиях усиливать водоудерживающую функцию. Однако здесь трудно выделить какие-либо общие свойства. Каждый тип белка проявляет себя по разному в зависимости от его состава, структуры и конформации. Таким образом, влияние температуры на белок требует тщательного изучения в каждом конкретном случае.

\section{2. Описание модели}

В настоящей работе разработана математическая модель на основе работ [1-3] влияния температуры на характер связывания белковых димеров в водном растворе. В качестве модельных систем нами были взяты гистоновые димеры Н2А-Н2B и Н3-H4. Структуры выбранных белков Н2А, Н2B, Н3 и Н4 представлены на рис. 1.

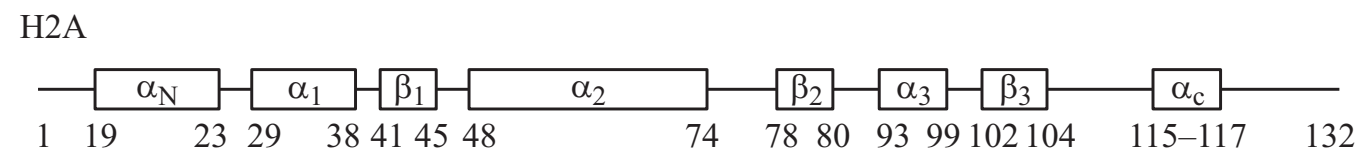

H2B



$\mathrm{H} 3$

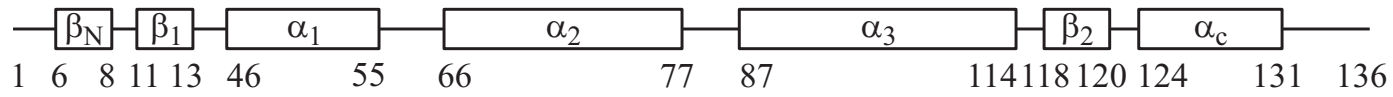

$\mathrm{H} 4$

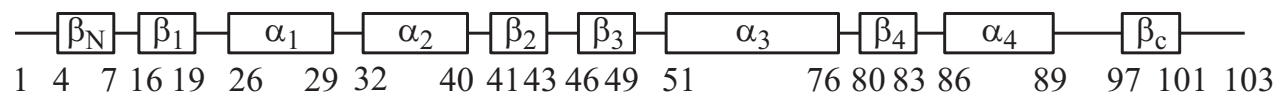

Рис. 1. Структуры гистоновых белков Н2А, Н2В, Н3, Н4. 
Таблица 1. Значение заряда $(Q)$ аминокислотного остатка (А.a) при конкретных значениях температуры

\begin{tabular}{|c|c|c|c|c|c|c|c|c|}
\hline \multirow{2}{*}{\multicolumn{2}{|c|}{$\begin{array}{c}\text { Амино- } \\
\text { кислотный } \\
\text { остаток }\end{array}$}} & \multicolumn{7}{|c|}{ Температура воды, ${ }^{\circ} \mathrm{C}$} \\
\hline & & \multirow{2}{*}{$\frac{20}{2.43 \cdot 10^{-19}}$} & \multirow{2}{*}{$\frac{25}{2.54 \cdot 10^{-19}}$} & \multirow{2}{*}{$\frac{30}{2.50 \cdot 10^{-19}}$} & \multirow{2}{*}{$\frac{35}{2.44 \cdot 10^{-19}}$} & \multirow{2}{*}{$\frac{40}{2.29 \cdot 10^{-19}}$} & \multirow{2}{*}{$\frac{45}{2.08 \cdot 10^{-19}}$} & \multirow{2}{*}{$\frac{50}{1.81 \cdot 10^{-19}}$} \\
\hline 1 & $A$ & & & & & & & \\
\hline 2 & $R$ & $-1.62 \cdot 10^{-19}$ & $-1.46 \cdot 10^{-19}$ & $-1.44 \cdot 10^{-19}$ & $-1.41 \cdot 1-^{-19}$ & $-1.39 \cdot 10^{-19}$ & $-1.31 \cdot 10^{-19}$ & $-1.23 \cdot 10^{-19}$ \\
\hline 3 & $N$ & $0.30 \cdot 10^{-19}$ & $0.16 \cdot 10^{-19}$ & $0.12 \cdot 10^{-19}$ & $0.13 \cdot 10^{-19}$ & $0.16 \cdot 10^{-19}$ & $0.29 \cdot 10^{-19}$ & $0.44 \cdot 10^{-19}$ \\
\hline 4 & $D$ & $-0.015 \cdot 10^{-19}$ & $-0.13 \cdot 10^{-19}$ & $-0.16 \cdot 10^{-19}$ & $-0.22 \cdot 10^{-19}$ & $-0.29 \cdot 10^{-19}$ & $-0.30 \cdot 10^{-19}$ & $-0.36 \cdot 10^{-19}$ \\
\hline 5 & $C$ & $3.46 \cdot 10^{-19}$ & $3.58 \cdot 10^{-19}$ & $3.54 \cdot 10^{-19}$ & $3.45 \cdot 10^{-19}$ & $3.25 \cdot 10^{-19}$ & $2.95 \cdot 10^{-19}$ & $2.59 \cdot 10^{-19}$ \\
\hline 6 & $Q$ & $-1.06 \cdot 10^{-19}$ & $-1.12 \cdot 10^{-19}$ & $-1.11 \cdot 10^{-19}$ & $-1.04 \cdot 10^{-19}$ & $-0.97 \cdot 10^{-19}$ & $0.92 \cdot 10^{-19}$ & $0.788 \cdot 10^{-19}$ \\
\hline 7 & $\tilde{E}$ & $0.16 \cdot 10^{-19}$ & $-0.20 \cdot 10^{-19}$ & $-0.23 \cdot 10^{-19}$ & $-0.31 \cdot 10^{-19}$ & $-0.39 \cdot 10^{-19}$ & $0.51 \cdot 10^{-19}$ & $-0.65 \cdot 10^{-19}$ \\
\hline 8 & $G$ & $1.71 \cdot 10^{-19}$ & $1.70 \cdot 10^{-19}$ & $1.77 \cdot 10^{-19}$ & $1.68 \cdot 10^{-19}$ & $1.59 \cdot 10^{-19}$ & $1.47 \cdot 10^{-19}$ & $1.33 \cdot 10^{-19}$ \\
\hline 9 & $H$ & $0.082 \cdot 10^{-19}$ & $0.16 \cdot 10^{-19}$ & $0.194 \cdot 10^{-19}$ & $0.193 \cdot 10^{-19}$ & $0.17 \cdot 10^{-19}$ & $0.19 \cdot 10^{-19}$ & $0.197 \cdot 10^{-19}$ \\
\hline 10 & $I$ & $1.52 \cdot 10^{-19}$ & $1.53 \cdot 10^{-19}$ & $1.50 \cdot 10^{-19}$ & $1.45 \cdot 10^{-19}$ & $1.32 \cdot 10^{-19}$ & $1.21 \cdot 10^{-19}$ & $1.04 \cdot 10^{-19}$ \\
\hline 11 & $L$ & $1.23 \cdot 10^{-19}$ & $1.24 \cdot 10^{-19}$ & $1.23 \cdot 10^{-19}$ & $1.19 \cdot 10^{-19}$ & $1.12 \cdot 10^{-19}$ & $0.97 \cdot 10^{-19}$ & $0.79 \cdot 10^{-19}$ \\
\hline 12 & $K$ & $-1.99 \cdot 10^{-19}$ & $-1.73 \cdot 10^{-19}$ & $-1.83 \cdot 10^{-19}$ & $-1.79 \cdot 10^{-19}$ & $-1.78 \cdot 10^{-19}$ & $-1.74 \cdot 10^{-19}$ & $-1.68 \cdot 10^{-19}$ \\
\hline 13 & $M$ & $0.41 \cdot 10^{-19}$ & $0.48 \cdot 10^{-19}$ & $0.51 \cdot 10^{-19}$ & $0.44 \cdot 10^{-19}$ & $0.38 \cdot 10^{-19}$ & $0.32 \cdot 10^{-19}$ & $0.22 \cdot 10^{-19}$ \\
\hline 14 & $F$ & $1.26 \cdot 10^{-19}$ & $1.32 \cdot 10^{-19}$ & $1.19 \cdot 10^{-19}$ & $1.24 \cdot 10^{-19}$ & $1.11 \cdot 10^{-19}$ & $0.93 \cdot 10^{-19}$ & $0.67 \cdot 10^{-19}$ \\
\hline 15 & $P$ & $0.45 \cdot 10^{-19}$ & $0.67 \cdot 10^{-19}$ & $0.29 \cdot 10^{-19}$ & $0.26 \cdot 10^{-19}$ & $0.14 \cdot 10^{-19}$ & $0.015 \cdot 10^{-19}$ & $-0.29 \cdot 10^{-19}$ \\
\hline 16 & $S$ & $0.76 \cdot 10^{-19}$ & $0.83 \cdot 10^{-19}$ & $0.82 \cdot 10^{-19}$ & $0.88 \cdot 10^{-19}$ & $0.84 \cdot 10^{-19}$ & $0.81 \cdot 10^{-19}$ & $0.77 \cdot 10^{-19}$ \\
\hline 17 & $T$ & $1.26 \cdot 10^{-19}$ & $1.32 \cdot 10^{-19}$ & $1.30 \cdot 10^{-19}$ & $1.28 \cdot 10^{-19}$ & $1.23 \cdot 10^{-19}$ & $1.15 \cdot 10^{-19}$ & $1.11 \cdot 10^{-19}$ \\
\hline 18 & $W$ & $1.24 \cdot 10^{-19}$ & $1.25 \cdot 10^{-19}$ & $1.15 \cdot 10^{-19}$ & $1.00 \cdot 10^{-19}$ & $0.77 \cdot 10^{-19}$ & $0.52 \cdot 10^{-19}$ & $0.107 \cdot 10^{-19}$ \\
\hline 19 & $Y$ & $0.87 \cdot 10^{-19}$ & $0.83 \cdot 10^{-19}$ & $0.86 \cdot 10^{-19}$ & $0.75 \cdot 10^{-19}$ & $0.63 \cdot 10^{-19}$ & $0.49 \cdot 10^{-19}$ & $0.20 \cdot 10^{-19}$ \\
\hline 20 & $V$ & $1.24 \cdot 10^{-19}$ & $1.30 \cdot 10^{-19}$ & $1.29 \cdot 10^{-19}$ & $1.27 \cdot 10^{-19}$ & $1.2 \cdot 10^{-19}$ & $1.11 \cdot 10^{-19}$ & $0.98 \cdot 10^{-19}$ \\
\hline
\end{tabular}

Отметим, что гистоновые белки являются основными белками, которые образуют октамер нуклеосомы ДНК. Каждый из белков представлен в нуклеосоме ДНК дважды. Восемь гистоновых белков нуклеосомы собраны в четыре гетеродимера: два Н2А-Н2В и два Н3-H4.

Для анализа стабильности димеров в водном растворе при увеличении температуры от 20 до $50^{\circ} \mathrm{C}$ мы выполнили расчет матрицы потенциальной энергии электростатического взаимодействия между различными аминокислотными остатками, при этом были сделаны следующие предположения.

1. Каждый аминокислотный остаток взаимодействует со всеми остальными аминокислотными остатками с определенным зарядом (см. табл. 1).

2. Для аминокислотных последовательностей, которые имеют различную длину, мы предположили, что оставшиеся от более длинной полипептидной последовательности непарные аминокислотные остатки могут взаимодействовать с собственной аминокислотной последовательностью. Поэтому мы дополнили более короткие аминокислотные последовательности аминокислотными остатками со средним значением кулоновского заряда, равным $0.45 \cdot 10^{-19} \mathrm{C}[1]$.

Для получения заряда для каждого аминокислотного остатка с учетом первого предположения мы использовали данные из работы [7], в которой авторы получили потенциал в зависимости от температуры для конкретной аминокислоты.
Отметим, что при увеличении температуры у различных белков наблюдается сложная зависимость изменения объема, поскольку в процессе нагрева могут происходить различные нарушения трехмерной структуры, которые отличаются по характеру у различных белков [10,11]. Мы взяли данные из [11], в которой приведены белки с известной аминокислотной последовательностью (Conalbumin, BSA, Hemoglobin, Ovalbumin,Pepsin, $\alpha$-Chymotrypsinogen A, $\alpha$-Chymotrypsin, Trypsin, Trypsinogen, $\beta$-Lactoglobulin, Myoglobin, $\alpha$-Lactalbumin, Lysozyme, Ribonuclease A, Cytochrome), а также даны их объемы при конкретном значении температуры. Отсюды мы рассчитали порядок величины $\delta$, на которую может изменяться радиус каждого аминокислотного остатка при изменении температуры на $5^{\circ} \mathrm{C}$.

Поскольку увеличение объема белка происходит неравномерно, то в нашей модели были сделаны следующие предположения.

1. Радиус гидрофобных аминокислотных остатков $(A, I, L, M, F, P, W, Y, V)$ уменьшается на величину $\delta$ при увеличении температуры на каждые $5^{\circ} \mathrm{C}$.

2. Радиус остальных аминокислотных остатков увеличивается на величину $\delta$ при увеличении температуры на каждые $5^{\circ} \mathrm{C}$.

Начальные радиусы аминокислот были взяты из [1] и в зависимости от типа аминокислотного остатка мы получили новые радиусы аминокислотных остатков 
Таблица 2. Изменение радиуса $(R)$ аминокислотных остатков (А.a)

\begin{tabular}{c|c|c|c|c|c|c|c}
\hline$A . a$ & $A$ & $R$ & $N$ & $D$ & $C$ & $Q$ & $E$ \\
\hline$R, n m$ & $0.60-\delta$ & $0.809+\delta$ & $0.68+\delta$ & $0.66+\delta$ & $0.629+\delta$ & $0.725+\delta$ & $0.714+\delta$ \\
\hline$A . a$ & $G$ & $H$ & $I$ & $L$ & $K$ & $M$ & $F$ \\
\hline$R, n m$ & $0.725+\delta$ & $0.732+\delta$ & $0.735-\delta$ & $0.734-\delta$ & $0.737+\delta$ & $0.74-\delta$ & $0.78-\delta$ \\
\hline$A . a$ & $P$ & $S$ & $T$ & $W$ & $Y$ & $V$ & - \\
\hline$R, n m$ & $0.672-\delta$ & $0.615+\delta$ & $0.659+\delta$ & $0.826-\delta$ & $0.78-\delta$ & $0.694-\delta$ & -
\end{tabular}

Таблица 3. Значения коэффициента $\delta$ при различных температурах

\begin{tabular}{c|c|c|c|c|c|c|c}
\hline${\text { Температура воды, }{ }^{\circ} \mathrm{C}}^{2}$ & 20 & 25 & 30 & 35 & 40 & 45 & 50 \\
\hline$\delta, \mathrm{nm}$ & 0 & $\delta$ & $2 \delta$ & $3 \delta$ & $4 \delta$ & $5 \delta$ & $6 \delta$
\end{tabular}

с учетом температуры водного окружения. При этом $\delta=4.6 \cdot 10^{-4} \mathrm{~nm}$.

Таким образом, каждому значению температуры водного раствора соответствует свой набор из 20 радиусов аминокислотных остатков.

Для получения расстояния между аминокислотными остатками мы воспользовались ранее выдвинутыми предположениями [1].

1. Между разноименными заряженными аминокислотными остатками расстояние было принято $0.15 \mathrm{~nm}$.

2. Между одноименно заряженными аминокислотными остатками расстояние было принято $0.4 \mathrm{~nm}$.

3. Расстояние между аминокислотными остатками, образующими, предположительно, одну водородную связь, было принято $0.35 \mathrm{~nm}$.

4. Расстояние между аминокислотными остатками, которые, предположительно, могут образовывать более одной водородной связи, было выбрано $0.25 \mathrm{~nm}$.

5. Расстояние между остальными аминокислотными остатками было принято $0.3 \mathrm{~nm}$.

Эти предположенияя мы дополнили следующим образом.

1. Мы выделили некоторые гидрофобные аминокислотные остатки $(I, V, L, F, W, M, A, Y)$ из общего числа аминокислотных остатков, между которыми было установлено расстояние $0.36 \mathrm{~nm}[12,13]$.

2. Мы допустили, что взаимодействие между гидрофобными аминокислотными остатками ослабевает с увеличением температуры, тогда расстояние будет уменьшаться на величину $2 \delta$.

3. Расстояние между остальными аминокислотными остатками увеличивается на величину $\delta$ при увеличении температуры на каждые $5^{\circ} \mathrm{C}$ (см. табл. 2 и 3 ).

Отметим, что пролин $(P)$ является уникальной аминокислотой, поскольку группа NH пептидной цепи входит в состав пятичленного кольца аминокислоты, поэтому в нашей модели имеет особые свойства, отличные от гидрофобных аминокислот и от всех остальных аминокислот. Так, его объем в нашей модели уменьшается на величину $\delta$ каждые $5^{\circ} \mathrm{C}$, при этом расстояние при взаимодействии с остальными аминокислотными остатками меняется, как у негидрофобных аминокислот, на величину $(0.3+\delta) \mathrm{nm}$.

Поскольку каждой температуре водного раствора соответствует свое значение электрической проницаемости, значение для всех температур, используемых в наших расчетах, мы свели в табл. 4.

Для анализа процессов влияние температуры на характер связывания димеров белков в водных растворах мы используем понятие числа обусловленности [1,2], которое будет характеризовать в данной физической постановке степень устойчивости конфигурации биологического комплекса при различных температурных режимах.

\section{3. Численное моделирование влияния температуры на характер связывания мономерных белков в водных растворах}

Для численного моделирования влияния температуры на характер связывания мономерных белков в димеры в водных растворах были выбраны небольшие белки, у которых длины аминокислотных последовательностей не сильно отличались. Нами были взяты последовательности гистоновых белков Н2А, Н2B, Н3, Н4 из базы данных [15], номера в базе данных составили соответветственно: Р04911, Р02293, Р61830, Р02309.

Отметим, что при повышении температуры будет увеличиваться энергия теплового движения, что может приводить к разрушению сольватных оболочек и соответственно к агрегированию системы. При агрегировании мы считаем, что происходит более сильное электростатическое взаимодействие между аминокислотными остатками, и это должно приводить к резкому снижению величины $\lg (\operatorname{cond}(W))$, где cond $(W)$ - число обусловленности матрицы потенциальной энергии электростатического взаимодействия, которая определяется 
Таблица 4. Значение относительной диэлектрической проницаемости воды при различных температурах воды [14]

\begin{tabular}{c|c|c|c|c|c|c|c}
\hline Температура воды, ${ }^{\circ} \mathrm{C}$ & 20 & 25 & 30 & 35 & 40 & 45 & 50 \\
\hline Диэлектрическая проницаемость воды & 80.103 & 78.304 & 76.546 & 74.828 & 73.151 & 71.512 & 69.910
\end{tabular}

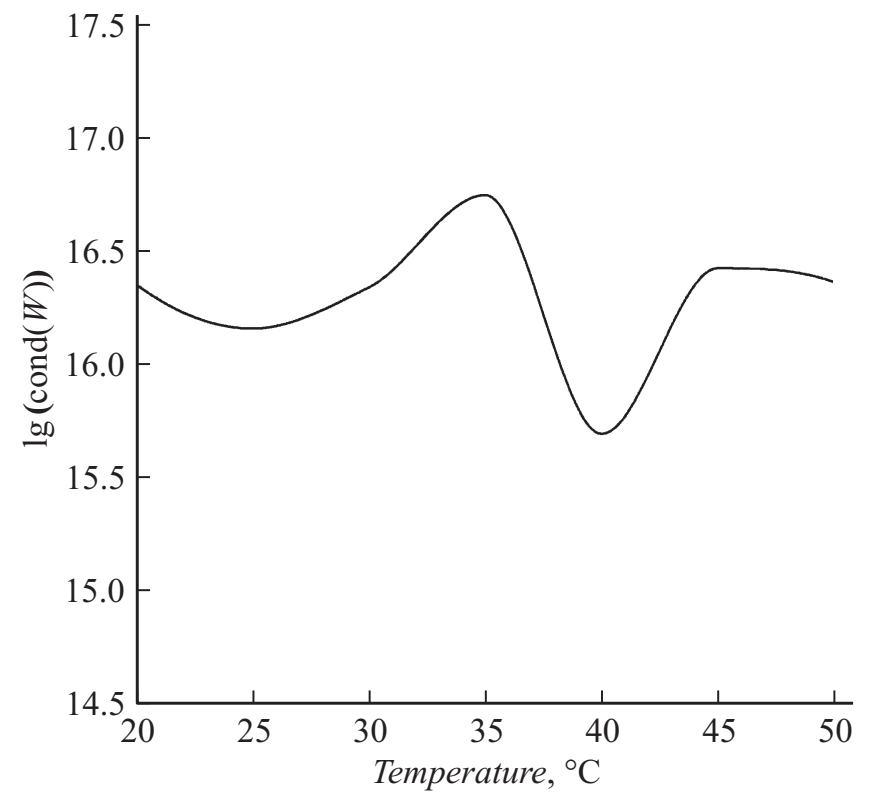

Рис. 2. Зависимость $\lg (\operatorname{cond}(W))$ от температуры для димера $\mathrm{H} 2 \mathrm{~A}-\mathrm{H} 2 \mathrm{~B}$.

следующим образом:

$$
\operatorname{cond}\left(W_{k}\right)=\left\|W_{k}\right\|-\left\|W_{k}^{-1}\right\| .
$$

$\left\|W_{k}\right\|$ - норма матрицы потенциальной энергии попарного электростатического взаимодействия между исследуемыми белками, $k$ - число матриц. При этом для выбора более устойчивого биохимического соединения между белками мы выбираем матрицу потенциальной энергии электростатического взаимодействия с наименьшим значением числа обусловленности [2].

На рис. 2 и 3 представлена температурная зависимость $\lg (\operatorname{cond}(W))$ для выбранных нами димеров $\mathrm{H} 2 \mathrm{~A}-\mathrm{H} 2 \mathrm{~B}$ и $\mathrm{H} 3-\mathrm{H} 4$.

Рассмотрим отдельно пики при различных температуpax для димера Н2А-Н2В. Как мы видим при увеличении температуры водного раствора от 20 до $50^{\circ} \mathrm{C}$, димер H2A-H2B демонстрирует сложное поведение в водном растворе как минимум двумя структурными переходами, максимальный переход приходится на температуру в области $35^{\circ} \mathrm{C}$. Начальное значение величины $\lg (\operatorname{cond}(W))$ при $20^{\circ} \mathrm{C}$ составило 16.4 , при температуре $35^{\circ} \mathrm{C}$ величина $\lg (\operatorname{cond}(W))$ повышается до значения 16.7 , достигая минимального значения в области $40^{\circ} \mathrm{C}$, равного 15.73 .

Как известно, белки являются полиэлектролитами и состоят из различных аминокислотных остатков, взаимодействия между которыми определяют структурные и ферментативные особенности каждого белка. Взаимодействие между аминокислотами осуществляется при участии сил различной природы (электростатические, гидрофобные, водородные, ковалентные), что в свою очередь объясняет повышенную по сравнению с нейтральными полимерами склонность цепей полиэлектролитов к конформационным изменениям при изменении температуры раствора, природы растворителя. Конформационное изменение участка цепи вызывает структурное изменение соседних звеньев цепи. Таким образом, возможное изменение конформации в цепи одного остатка затрагивает положение соседних аминокислотных остатков, изменение положения которых влечет за собой изменение в положении соседствующих с ними последующих аминокислотных остатков. Если молекула белка может существовать в нескольких энергетических конформациях, то возможна ситуация, когда изменение в распределении зарядов вызывает резкий переход из одного конформационного состояния в другое.

Таким образом, при увеличении температуры мы предполагаем, что наблюдаются конформационные переходы, что приводит к уменьшению связанности мономерных субъединиц Н2А и Н2В в диапазоне температур от 20 до $35^{\circ} \mathrm{C}$ и от 40 до $50^{\circ} \mathrm{C}$. Увеличение величины $\lg (\operatorname{cond}(W))$ говорит об уменьшении связывания димера H2A-H2B в водном растворе в выбранном температурном диапазоне. Незначительное уменьшение значения

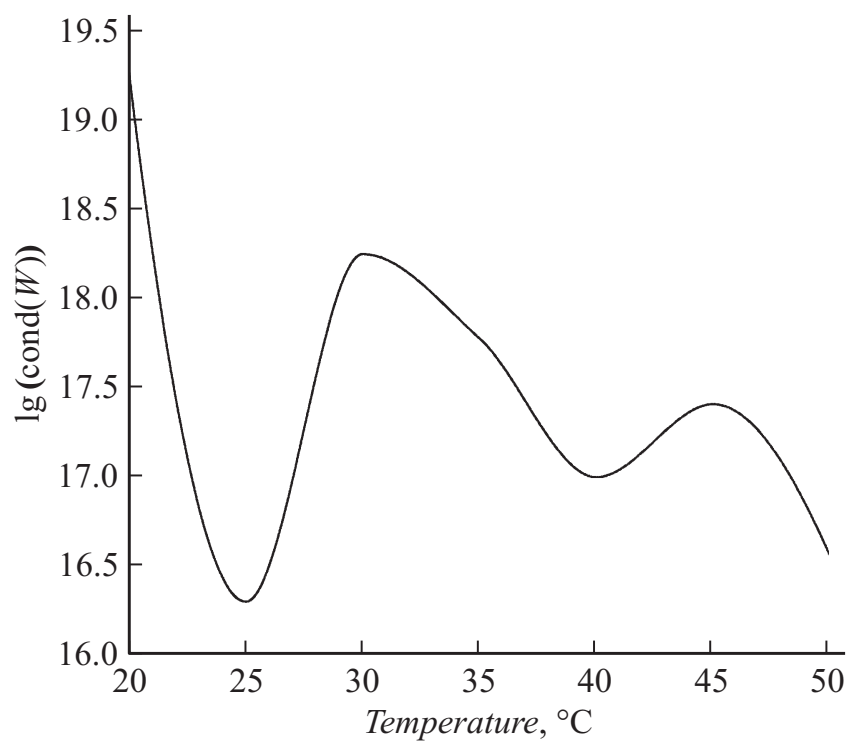

Рис. 3. Зависимость $\lg (\operatorname{cond}(W))$ от температуры для димера H3-H4. 
$\lg (\operatorname{cond}(W))$ в диапазоне от 35 до $40^{\circ} \mathrm{C}$ указывает на отсутствие агрегирования димера.

Полученные результаты математического моделирования и их последующий анализ хорошо согласуются с результатами $[16,17]$, в которых указывается на отсутствие агрегации для димера Н2А-Н2B в заданном диапазоне температур.

Результаты же, полученные для димера Н3-H4, наоборот, демонстрируют склонность к образованию агрегатов, поскольку $\lg (\operatorname{cond}(W))$ уменьшается от значения 19.3 до величины 16.25 при увеличении температуры от 20 до $25^{\circ} \mathrm{C}$ с двумя „плечами“ в области 30 и $45^{\circ} \mathrm{C}$.

Анализ полученных результатов позволяет нам предположить возможность агрегации, а также формирования новых сложных структур с конформационными переходами димера Н3-H4 в выбранном температурном диапазоне.

Наблюдаемый максимальный пик в области $30^{\circ} \mathrm{C}$ мы объясняем структурными изменениями, что в свою очередь влияет на связывания различных аминокислотных остатков. Полученные результаты для димера Н3-H4 находятся в хорошем согласии с ранее выполненной экспериментальной работой [18], в которой указывается, что белки Н3 и Н4 имеют склонность образовывать агрегаты в водных растворах.

Также в нашей работе было выполнено моделирование связывания различных участков вторичной структуры ( $\alpha$-спирали и $\beta$-слои $)$ гистоновых белков $\mathrm{H} 2 \mathrm{~A}-\mathrm{H} 2 \mathrm{~B}$ и $\mathrm{H} 3-\mathrm{H} 4$ и проанализирована их тенденция к образованию агрегатов. Нами были выбраны следующие участки белковых последовательно-

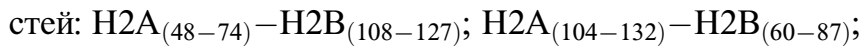
$\mathrm{H} 3_{(46-77)}-\mathrm{H} 4_{(25-51)} ; \mathrm{H} 3_{(87-114)}-\mathrm{H} 4_{(83-103)}$, и мы построили для них графики зависимости величины $\lg (\operatorname{cond}(W))$ от температуры. Отметим, что в представленной работе были выбраны произвольные участки гистоновых белков, которые имеют известную вторичную структуру, представленную на рис. 1 , и проведен анализ их возможного взаимодействия в водных растворах при различной температуре.

Так, график на рис. 4 для последовательности $\mathrm{H} 2 \mathrm{~A}_{(48-74)}-\mathrm{H} 2 \mathrm{~B}_{(108-127)}$ имеет максимальный пик значения величины $\lg (\operatorname{cond}(W))$, равной 10.2 в области температуры $40^{\circ} \mathrm{C}$. Отметим, что величина пика при $40^{\circ} \mathrm{C}$ значительно отличается от значения величины $\lg (\operatorname{cond}(W))$ при начальной температуре исследований $20^{\circ} \mathrm{C}$, которая составила 9.15. Также мы наблюдаем два минимума на кривой температурной зависимости поведения белковой последовательности при температурах 30 и $45^{\circ} \mathrm{C}$.

Следует отметить, что минимальным из них является пик в области температуры $30^{\circ} \mathrm{C}$, значения же величины $\lg (\operatorname{cond}(W))$ при температуре $45^{\circ} \mathrm{C}$ несколько выше и составили 8.3 и 8.9 соответственно.

Таким образом, можно сделать вывод, основываясь на характере графика, что гистоновая последовательность

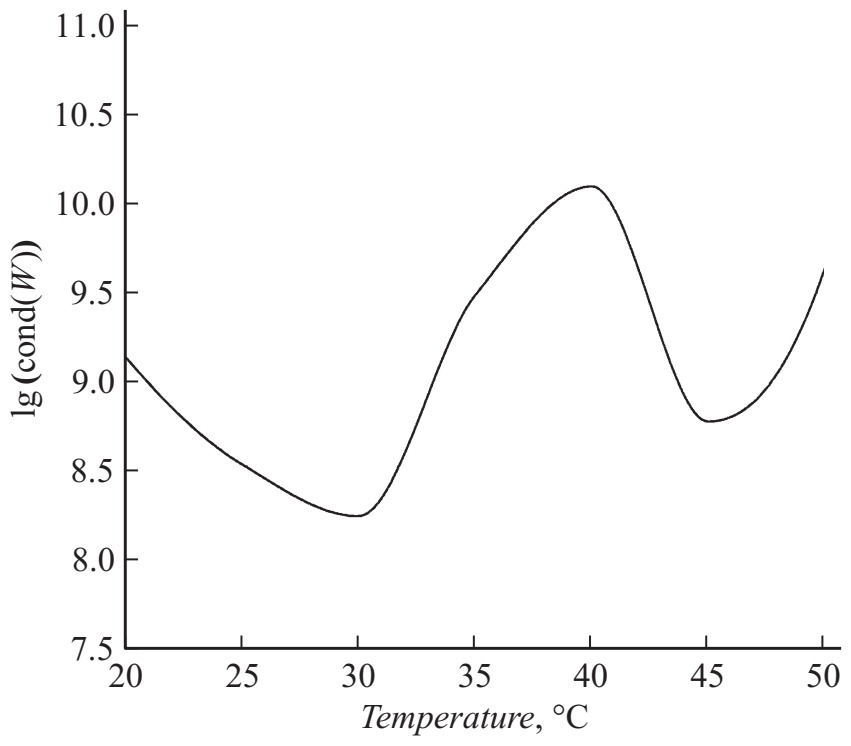

Рис. 4. Зависимость $\log (\operatorname{cond}(W))$ от температуры для доменов $\mathrm{H}_{2} \mathrm{~A}_{(48-74)}-\mathrm{H}_{2} \mathrm{~B}_{(108-127)}$.

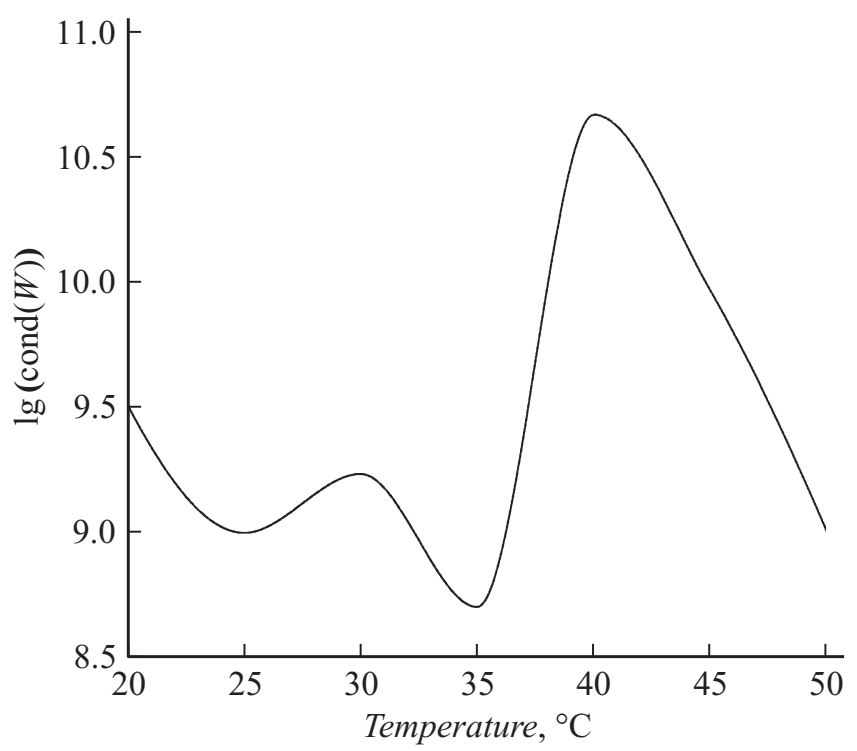

Рис. 5. Зависимость $\lg (\operatorname{cond}(W))$ от температуры для доменов $\mathrm{H} 2 \mathrm{~A}_{(104-132)}-\mathrm{H} 2 \mathrm{~B}_{(60-87)}$.

$\mathrm{H} \mathrm{A}_{(48-74)}-\mathrm{H}_{2} \mathrm{~B}_{(108-127)}$ не имеет склонности образовывать агрегаты в заданном температурном диапазоне, поскольку величина $\lg (\operatorname{cond}(W))$ имеет тенденцию к увеличению.

Рассмотрим рис. 5, на котором изображена температурная зависимость поведения белковой последовательности $\mathrm{H}_{2} \mathrm{~A}_{(104-132)}-\mathrm{H} 2 \mathrm{~B}_{(60-87)}$. Как мы видим из приведенного графика, зависмость величины $\lg (\operatorname{cond}(W))$ от температуры имеет сложный характер от 20 до $50^{\circ} \mathrm{C}$, достигая макимального значения 10.7 в области $40^{\circ} \mathrm{C}$.

Также на графике присутствует небольшой спад величины в диапазоне температур от 20 до $35^{\circ} \mathrm{C}$, при кото- 


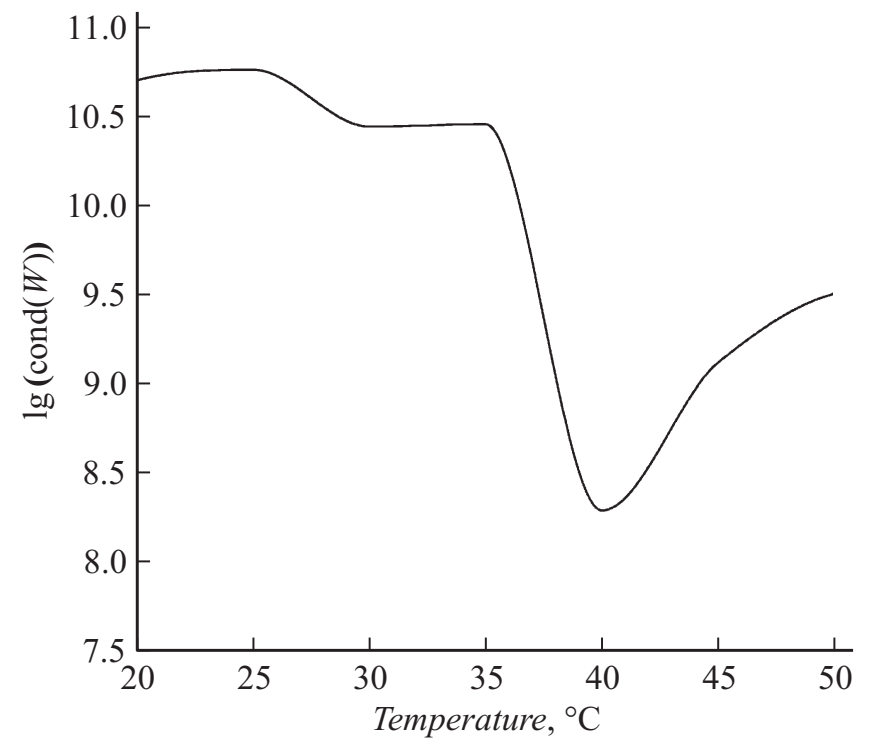

Рис. 6. Зависимость $\log (\operatorname{cond}(W))$ от температуры для доменов $\mathrm{H} 3_{(46-77)}-\mathrm{H} 4_{(25-51)}$.

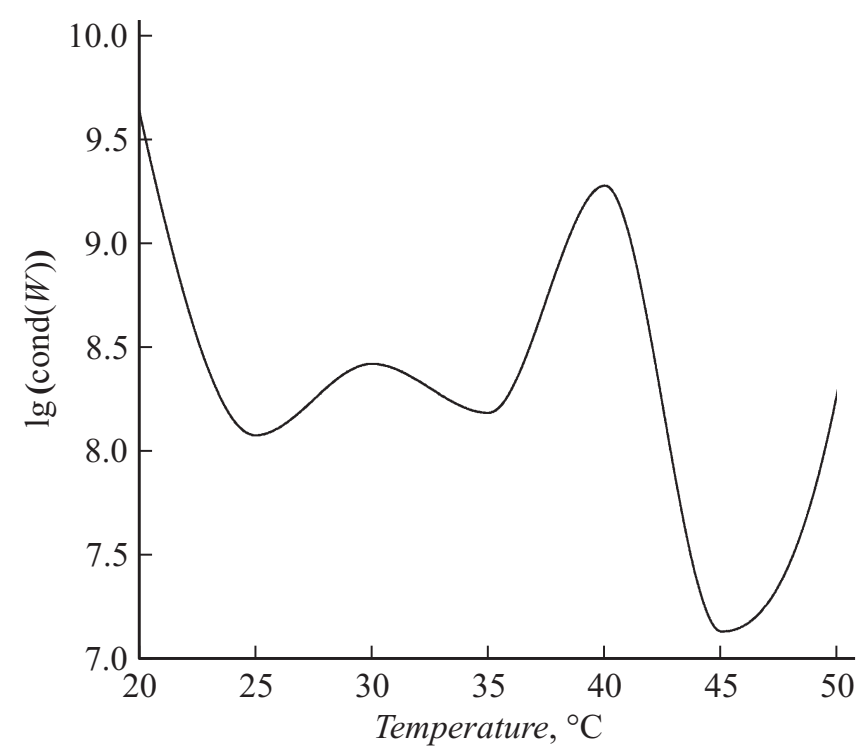

Рис. 7. Зависимость $\log (\operatorname{cond}(W))$ от температуры для доменов $\mathrm{H} 3_{(87-114)}-\mathrm{H} 4_{(83-103)}$.

рых значение величины $\lg (\operatorname{cond}(W))$ падает от 9.5 до 8.7 . В целом из характера графика можно сделать вывод, что взаимодействие данных аминокислотных участков усиливается в диапазоне температур от 20 до $35^{\circ} \mathrm{C}$ и значительно ослабевает в диапазоне температур от 35 до $40^{\circ} \mathrm{C}$, таким образом, в выбранном диапазоне температур мы можем предположить уменьшение связывания мономерных субьединиц в димере.

Перейдем к анализу взаимодействия гистоновых последовательностей белков Н3 и Н4. Рассмотрим более детально последовательность $\mathrm{H} 3_{(46-77)}-\mathrm{H} 4_{(25-51)}$, которая представлена на рис. 6. Как мы видим, на графике присутствует минимальный пик величины $\lg (\operatorname{cond}(W))$ при температуре в области $40^{\circ} \mathrm{C}$, которая достигает значения 8.25. Отметим, что максимальное значение величины $\lg (\operatorname{cond}(W))$ в области $20^{\circ} \mathrm{C}$ составляет 10.7. Таким образом, разница между максимальным и минимальным значениями величины $\lg (\operatorname{cond}(W))$ составила 2.45 . Подобное резкое снижение величины $\lg (\operatorname{cond}(W))$ в диапазоне температур от 35 до $40^{\circ} \mathrm{C}$ в данной постановке задачи мы трактуем как склонность белковых последовательностей к образованию агрегатов, что влечет за собой изменение конформации. Дальнейшее повышение величины $\lg (\operatorname{cond}(W))$ в диапазоне температур от 40 до $50^{\circ} \mathrm{C}$ также объясняется структурными перестройками при увеличении температуры.

Интересными являются результаты на рис. 7 для белковой последовательности $\mathrm{H} 3_{(87-114)}-\mathrm{H} 4_{(83-103)}$. На представленном графике мы видим несколько пиков. Так, два минимума наблюдаются в области температур 25 и $45^{\circ} \mathrm{C}$, которым отвечают значения величины $\lg (\operatorname{cond}(W)) 8.07$ и 7.1 соответственно. Два максимальных значениям при температурах 20 и $40^{\circ} \mathrm{C}$ отвечают значениям величины $\lg (\operatorname{cond}(W)) 9.6$ и 9.35 соответственно, при этом стоит отметить, что максимальное значение величина $\lg (\operatorname{cond}(W))$ принимает при начальной температуре исследования $20^{\circ} \mathrm{C}-9.6$ и на всех остальных температурах выше этого значения уже не поднимается. Разница в значениях величины $\lg (\operatorname{cond}(W))$ между начальной температурой и при температурах 25 и $45^{\circ} \mathrm{C}$ составила соответственно 1.53 и 2.5. Общий вид графика для последовательности $\mathrm{H} 3_{(87-114)}-\mathrm{H} 4_{(83-108)}$ имеет вид нисходящий кривой с пиками на различных температурах, что позволяет сделать вывод о склонности к увеличению взаимодействия между различными аминокислотными остатками и, как следствие, к возможному агрегированию.

Приведенный выше анализ кривых температурных зависимостей величины $\lg (\operatorname{cond}(W))$ для различных участков белков $\mathrm{H} 2 \mathrm{~A}, \mathrm{H} 2 \mathrm{~B}, \mathrm{H} 3$ и $\mathrm{H} 4$ указывает на более сильное взаимодействие аминокислотных последовательностей для белков НЗ и Н4 по сравнению со взаимодействием аминокислотных последовательностей гистоновых белков Н2А и Н2В.

Отметим также, что общий анализ кривых выбранных участков белков Н2А, Н2B, Н3 и Н4 совпадает с анализом для полных последовательностей гетеродимеров $\mathrm{H} 2 \mathrm{~A}-\mathrm{H} 2 \mathrm{~B}$ и $\mathrm{H} 3-\mathrm{H} 4$.

Однако мы ничего не можем сказать о характере связывания других участков гистоновых белков, которые не были проанализированы в нашей работе.

\section{Заключение}

В настоящей работе было выполнено математическое моделирование температурного влияния на устойчивость белковых димеров $\mathrm{H} 2 \mathrm{~A}-\mathrm{H} 2 \mathrm{~B}$ и Н3-H4, а также 
некоторых участков аминокислотных последовательностей в водном растворе с учетом вклада гидрофобных аминокислотных остатков в формирование структуры димеров. Наша модель позволила выявить характер связывания димеров Н2А-H2B и Н3-H4 в условиях различной температуры водного раствора.

Мы ввели следующие критерии, которые указывают на способность целых белковых молекул заданного строения и длины аминокислотной последовательности образовывать агрегаты в водном растворе при повышении температуры от 20 до $50^{\circ} \mathrm{C}$.

Так, если разница величин $\lg (\operatorname{cond}(W))$ при температуре $20^{\circ} \mathrm{C}$ и в нижней точке пика на последующих температурах меньше значения 2.0, то мы считаем, что выбранные нами гистоновые белки не склонны к образованию агрегатов.

Если разница величин $\lg (\operatorname{cond}(W))$ при температуре $20^{\circ} \mathrm{C}$ и в нижней точке пика на последующих температуpax больше значения 2.0, то мы считаем, что выбранные биологические объекты могут образовывать агрегаты в заданном температурном диапазоне.

Таким образом, анализ полученных кривых для димера Н2А-Н2В указывает на отсутствие заметной агрегации в выбранном диапазоне температур, поскольку разница в значениях $\lg (\operatorname{cond}(W))$ при температуре $20^{\circ} \mathrm{C}$ и в точке минимума составила 0.67 .

Анализ полученных данных для димера Н3-H4 указывает на склонность выбранных биологических систем к образованию агрегатов с увеличением температуры, поскольку величина $\lg (\operatorname{cond}(W))$ демонстрирует резкое снижение от 20 до $50^{\circ} \mathrm{C}$, с максимальной разницей в значениях величины $\lg (\operatorname{cond}(W))$, равной 3.05 .

Результаты, полученные для взаимодействия различных участков гистоновых белков Н3 и Н4, указывают на возможную агрегацию в заданном диапазоне температур, поскольку величина $\lg (\operatorname{cond}(W))$ неравномерно уменьшается от начальной температуры исследования 20 до $50^{\circ} \mathrm{C}$ и разница в величинах $\lg (\operatorname{cond}(W))$ между начальным и минимальным значением для участков белковых последовательностей Н3-Н4 намного больше, чем эта же величина для белковых последовательностей Н2A-Н2B. Проведенное численное моделирование связывания различных участков гистоновых белков Н2А и Н2B, Н3 и Н4 позволило сделать выводы о различной силе взаимодействия между выбранными аминокислотными последовательностями.

Таким образом, разработанная нами математическая модель позволяет проводить анализ агрегации различных димеров в водном растворе при повышении температуры и прогнозировать склонность белковых комплексов к агрегированию.

Исследования в данной области в дальнейшем позволят создавать белки с заданными физическими свойствами, которые будут агрегировать или растворяться в зависимости от предъявляемых к ним требований.
Авторы выражают благодарность профессору кафедры „Физическая электроника“ Санкт-Петербургского политехнического университета Петра Великого доктору физ.-мат. наук А.П. Головицкому за консультации и конструктивное обсуждение результатов.

\section{Список литературы}

[1] Куликов К.Г., Кошлан Т.В. // ЖТФ. 2016. Т. 86. Вып. 10. C. $131-138$.

[2] Кошлан Т.В., Куликов К.Г. // ЖТФ. 2017. Т. 87. Вып. 4. C. 489-497.

[3] Кошлан Т.В., Куликов К.Г. // ЖТФ. 2017. Т. 87. Вып. 5. C. 665-6718.

[4] Fink A.L. // Fold. Des. 1998. Vol. 3. N 1. P. 923.

[5] Dijk E., Hoogeveen A., Abeln S. // PLOS Comput. Biol. P. 1-17. http://dx.doi.org/10.1371/journal.pcbi.1004277.

[6] Баркан Я.Г. Органическая химия. М.: Высшая школа, 1973. $552 \mathrm{c}$.

[7] Петров К.П. Методы биохимии растительных продуктов. Киев: Вища школа, 1978. 224 с.

[8] Ботвинник М.М. Белки. М.: ИЛ, 1956.

[9] Гауровити, Ф. Химия и биология белков. М.: ИЛ, 1953. $438 \mathrm{c}$.

[10] Akasaka K., Matsuki H. High Pressure Bioscience Basic Concepts, Applications and Frontiers. Germany: Springer, 2015. 789 p.

[11] Chalikian T.V., Totrov M., Abagyan R., Kenneth J. // J. Mol. Biol. 1996. Vol. 260. P. 588-603.

[12] Hua L., Zangi R., Berne B.J. // J. Phys. Chem. C. 2009. Vol. 113. P. 52445253

[13] Zangi R., Berne B.J. // J. Phys. Chem. B. 2008. Vol. 112. N 29. P. 8634-8644.

[14] Malmberg C.G., Maryott A.A. // J. Res. Nat. Bur. Stand. 1956. Vol. 56. N 1. P. 1-8.

[15] The Universal Protein Resource //http://www.uniprot.org/.

[16] Karantza V., Freire E., Moudrianakis E.N. // Biochem. 2001. Vol. 40. P. 13114-13123.

[17] Karantza V., Freire E., Moudrianakis E.N. // Biochem. 1995. Vol. 34. P. 5988-5996.

[18] Karantza V., Freire E., Moudrianakis E.N. // Biochem. 1996. Vol. 35. P. 2037-2046. 